Submitted to the Annals of Applied Statistics

\title{
SUPPLEMENT TO “THE IMPORTANCE OF DISTINCT MODELING STRATEGIES FOR GENE AND GENE-SPECIFIC TREATMENT EFFECTS IN HIERARCHICAL MODELS FOR MICROARRAY DATA"
}

\author{
By STEVen P. Lund And DAN NetTleton \\ Iowa State University
}

1. Evidence Supporting Need for Three-level Hierarchical Models. The $\mathrm{LN}^{3} \mathrm{MV}$ model parameter estimates for the DC3000 and Mouse Diet datasets provide further evidence of the presence of gene effects in real data. To demonstrate, we analyzed a wide variety of data simulated from two-level hierarchical models and examined values of $\hat{\sigma}_{\gamma}^{2}$ obtained from analysis with the $\mathrm{LN}^{3} \mathrm{MV}$ method. We simulated from the LNN and LNNMV models for several different values of $\sigma_{\tau}^{2}$. We simulated from the GG model for several values of $\alpha_{0}$ and, separately, for several values of $\nu *$. All other model parameters were fixed at the corresponding value estimated from the DC3000 dataset analysis. The experimental design and expression pattern structure used for these simulations was the same as for the simulations described in Section 5 of Lund and Nettleton (2012).

We also simulated from the HIV dataset under the structure of the LNNMV 
model. That is, $y_{j i}=\mu+\tau_{j G_{p(j)}(i)}+\epsilon_{j i}$ where $p(j)$ is the desired expression pattern for the $j$ th gene, $\mu$ represents the average expression of all genes and groups, $\tau_{j G_{p(j)}(i)}$ represents a random group effect for observations from the $G_{p(j)}(i)$ th group in the $j$ th gene, and $\epsilon_{j i}$ represents a random error. To generate the data for simulation $b$, we randomly ordered the genes and randomly picked 9 subjects from the pool of 23 subjects who were identical with regard to the factors considered in the HIV study. Let $\mathbf{y}^{(b)}$ be the resulting $8793 \times 9$ matrix of log-scale observations for simulation $b$. We constructed error terms by subtracting row averages of this matrix from their corresponding observations $\left(\epsilon_{j i}^{(b)}=y_{j i}^{(b)}-\bar{y}_{j}^{(b)}\right)$. We randomly constructed each group effect using the (scaled) difference between the average of a randomly sampled row and the overall average. That is, $\tau_{j G_{p(j)}^{(i)}}^{(b)}=c^{(b)}\left(\bar{y}_{j^{\prime} \cdot}^{(b)}-\bar{y}_{\cdot \cdot}^{(b)}\right)$ where $j^{\prime}$ is a randomly selected integer between 1 and 8793 and $c^{(b)}$ is a constant chosen to determine the scale of the group effects' distribution in simulation $b$. Finally, we constructed simulated data as $y_{j i}^{\operatorname{sim}(b)}=\bar{y}_{\cdot \cdot}^{(b)}+\tau_{j G_{p(j)}^{(i)}}^{(b)}+\epsilon_{j i}^{(b)}$ and used the first 5001 rows of the resulting matrix. This approach ensured that differences due to treatment effects are distributed identically to differences across genes, as in each of the two-level hierarchical models.

The simulation results presented in Figure 1 show the $\mathrm{LN}^{3} \mathrm{MV}$ model produced smaller estimates of $\sigma_{\gamma}^{2}$ for the data simulated without gene effects than for the actual datasets. There was very little variability among the estimates of $\sigma_{\gamma}^{2}$ for 
simulated datasets where $\hat{\sigma}_{\tau}^{2}$ is less than 0.5 . Also, $\hat{\sigma}_{\tau}^{2}$ was substantially less than $\hat{\sigma}_{\gamma}^{2}$ for every simulated dataset, but the opposite was true for both of the actual datasets. This evidence supports our belief that there are often substantial gene effects present in real microarray data, which are not appropriately accounted for by any of the two-level hierarchical models.

2. Simulation Study. We conduct a variety of simulations to assess the accuracy and power of the considered methods. By "accuracy," we refer to the property that for any given collection of genes the average posterior probability for each pattern should closely match the proportion of genes in the collection that follow the given pattern. By "power," we refer to a method's ability to detect differential expression. We prefer the method that creates the largest list of genes for a given PPDE threshold, provided that the method's ePPDEs are accurate.

To examine the effectiveness of the proposed methods, we conducted a series of simulations for an experimental setup with 3 experimental conditions ( 1 control and 2 treatments) each with 3 replicates. As with the DC3000 data analysis in the previous section, our expression patterns of interest compared each treatment with the control. Thus, the simulations have $2^{2}=4$ different expression patterns. Each simulation scenario was repeated 10 times.

2.1. Model Based and Data Based Simulation Studies. We simulated data from each of the five models (GG, LNN, LNNMV, $\mathrm{LN}^{3}$ and $\mathrm{LN}^{3} \mathrm{MV}$ ) using the model 
parameters reported for the DC3000 dataset in Table 1. In addition to these model based simulations, we also conducted simulations using an HIV mRNA expression dataset from the GEO website, named GDS1449. Simulations based on real data examine model performance in more realistic scenarios where data include dependence across genes and do not perfectly follow known distributions. The HIV dataset contained expression data for 8793 genes from each of 87 subjects, 23 of whom shared a common treatment group. For each simulation based on the HIV dataset, we randomly sampled 9 subjects from the subset of 23 and sampled 5001 genes from the 8793 total genes in the dataset. The treatment effect for the $m$ th group in the $j$ th gene was simulated independently and identically as $\tau_{m j}=t_{m j} 2\left(b_{m j}-.5\right)$ where $t_{m j} \stackrel{i i d}{\sim}$ gamma(shape $\alpha=1$, rate $\left.\beta=1.25\right)$ and $b_{m j} \stackrel{i i d}{\sim} \operatorname{Bernoulli}(.5)$. The treatment effects were added to the HIV data to create the desired expression patterns. When analyzed by the $\mathrm{LN}^{3} \mathrm{GV}$ method, the 20 datasets produced by this simulation method had parameter estimate averages (and standard errors) $\hat{\mu}=5.33(0.005), \hat{\sigma}_{\gamma}^{2}=1.539(0.022), \hat{\sigma}_{\tau}^{2}=0.960(0.016), \hat{\nu}=$ $3.284(0.027), \hat{\Phi}=0.255(0.004)$.

For every simulation, 3000 genes were simulated to follow the null pattern (all treatments are EE with the control) and 667 genes for each of the other expression patterns of interest. We then analyzed each simulated dataset with each method and recorded estimated posterior probabilities for each expression pattern for each 

gene.

2.2. Simulation Results. The LNN, LNNMV*, and LNNGV methods are special cases of the $\mathrm{LN}^{3}, \mathrm{LN}^{3} \mathrm{MV}^{*}$, and $\mathrm{LN}^{3} \mathrm{GV}$ models in which $\sigma_{\gamma}^{2}=0$. For data simulated from models without gene effects, we would expect estimated expression pattern posterior probabilities from the LNN, LNNMV*, and LNNGV methods to closely match those from the $\mathrm{LN}^{3}, \mathrm{LN}^{3} \mathrm{MV} *$, and $\mathrm{LN}^{3} \mathrm{GV}$ models, respectively. All correlations between the PPDEs estimated from the LNN, LNNMV*, and LNNGV methods and the PPDEs estimated from the $\mathrm{LN}^{3}, \mathrm{LN}^{3} \mathrm{MV} *$, and $\mathrm{LN}^{3} \mathrm{GV}$ methods, respectively, were greater than .998 for each of the 10 simulations from each of the GG, LNN, and LNNMV models. This demonstrates that the methods based on three-level hierarchical models can adapt to data without gene effects and perform nearly identically to two-level methods when data are generated from a two-level hierarchical model. Thus, our results indicate that there is no harm in using a threelevel method, even if the true data generating mechanism is consistent with a twolevel hierarchy. In contrast, many of the results that we shall present subsequently show that two-level methods can perform quite poorly relative to three-level methods when the true data generating mechanism is consistent with a three-level hierarchy.

For any given collection of genes, the average estimated posterior probability for each pattern should closely match the proportion of genes in the collection 
that follow the given pattern. We focus in particular on collections of genes that have been assigned small estimated posterior probabilities of equivalent expression (ePPEEs) as these are of primary interest in practice. To examine the methods with respect to this criterion, for each simulated dataset, we sorted genes according to their ePPEEs, from smallest to largest. Beginning with the 100 genes with the smallest ePPEEs, we created lists by adding genes with the smallest ePPEE one at a time and plotting the observed proportion of listed genes that were actually simulated from the null expression pattern versus the average ePPEE for the listed genes. An ideal method should produce curves which closely follow the $y=x$ diagonal. Curves appearing substantially above (below) the $y=x$ diagonal in the plotted range are considered liberal (conservative) relative to their reported posterior probability estimates.

Figure 2 displays results from applying each method to ten separate simulations from the HIV dataset and each of the five models. The plots for the LNNMV simulation indicate that methods using point estimates of gene-specific error variances (LNNMV, LNNMV*, and $\mathrm{LN}^{3} \mathrm{MV}^{*}$ ) produced liberal ePPEEs, as did methods assuming a constant coefficient of variation (GG, LNN, and $\left.\mathrm{LN}^{3}\right)$. EBarrays' LNNMV method was most liberal because it uses a biased gene-specific variance estimator that tends to underestimate each true variance. The LNNMV, LNNMV*, and $\mathrm{LN}^{3} \mathrm{MV}^{*}$ methods underestimated the proportion of null genes appearing on 
a gene list for several simulation models, including LNNMV. That is, EBarrays' LNNMV method produced liberal posterior probability estimates even when analyzing data simulated from its own model. Liberal posterior probability estimates lead to misplaced confidence in the underlying expression pattern for a given gene, which is problematic when creating gene lists with controlled error rates. The LNNGV and $\mathrm{LN}^{3} \mathrm{GV}$ curves closely follow the $y=x$ diagonal for the LNNMV simulations, which demonstrates the validity of our suggested method for handling gene-specific error variances.

The plots for the $\mathrm{LN}^{3}$ and $\mathrm{LN}^{3} \mathrm{MV}$ simulations indicate that methods omitting gene effects produced conservative ePPEEs when gene effects are present in the data. When analyzing data simulated from the $\mathrm{LN}^{3} \mathrm{MV}$ model, the LNNMV and LNNMV* methods suffer from two separate, counteracting issues: omitting gene effects, which leads to conservative ePPEEs, and using point estimates of genespecific error variances, which leads to liberal ePPEEs. With the exception of the HIV dataset simulations, the $\mathrm{LN}^{3} \mathrm{GV}$ curves closely follow the $y=x$ diagonal for all simulation scenarios, indicating this method produced robustly accurate ePPEEs.

There is substantial variability between the curves in each plot for simulations from the HIV dataset. It is, nonetheless, clear that the $\mathrm{LNNGV}$ and $\mathrm{LN}^{3} \mathrm{GV}$ methods were the only methods that did not produce blatantly liberal posterior probabil- 
ity estimates. The LNNGV appeared to be slightly conservative, while the $\mathrm{LN}^{3} \mathrm{GV}$ was not clearly biased.

We next examine the power of each method. A method that says all expression patterns are equally likely for every gene is not very useful. Assuming the posterior probability estimates are accurate as discussed above, a method that strongly differentiates among the expression patterns for as many genes as possible is preferable. That is, we would prefer the method that creates the largest list of genes for a given ePPEE threshold, provided that the method's ePPEEs are accurate. To examine the methods with respect to this criterion, we sorted genes according to their ePPDEs from smallest to largest. We then plotted the average ePPEE for each rank across the ten simulation iterations versus rank. (Averaging by rank allows us to create one plot for all simulation iterations and to use standard error lines, rather than requiring a separate plot for each simulation iteration or model.) These plots quickly provide the estimated size of a gene list for any ePPEE cutoff, given by intersecting a vertical line at the desired cutoff with the curves for each method. Figure 3 displays the average results for $\mathrm{LNNGV}, \mathrm{LN}^{3} \mathrm{GV}$, and LNNMV methods applied to ten simulations from the HIV dataset and the $\mathrm{LN}^{3} \mathrm{MV}$ model. Dashed lines are \pm two standard errors. There are substantial differences between the curves. While the LNNMV method tends to place more genes on a list for PPEE thresholds near zero, this method was demonstrated to give substantially liberal ePPEEs. In both 
cases, the $\mathrm{LN}^{3} \mathrm{GV}$ method places noticeably more genes on a DE list for a given threshold than does the LNNGV method.

Finally, we examine the significance rankings of each method by creating ROC curves. The solid curves in Figures 4-6 display ROC curves averaged across ten simulations from each of the models. The dashed lines are \pm two standard errors around the mean, providing approximate $95 \%$ confidence intervals. The ROC curves for the HIV, $\mathrm{LN}^{3} \mathrm{MV}$, and LNNMV simulations are widely separated into two groups with curves for methods assuming a constant coefficient of variation falling substantially beneath curves for methods allowing for gene-specific error variances. In the $\mathrm{LN}^{3}$ simulations, the ROC curves for the methods based on twolevel models fall slightly beneath curves for methods based on three-level models. The ROC curves for data simulated from the LNN and GG models show little difference between any of the methods, with the exception that the LNNMV method may produce a curve slightly lower than those of the other methods. No method produces ROC curves higher than those produced by the $\mathrm{LN}^{3} \mathrm{GV}$ method for any considered simulation. In general, these plots demonstrate that in addition to producing accurate, powerful ePPDEs, the $\mathrm{LN}^{3} \mathrm{GV}$ method also produces improved ROC curves that are robust to model mis-specification.

[Fig 1 about here.]

[Fig 2 about here.] 


\section{LUND AND NETTLETON}

[Fig 3 about here.]

[Fig 4 about here.]

[Fig 5 about here.]

[Fig 6 about here.]

\section{References.}

Lund, S. P. and Nettleton, D. (2012). The Importance of Distinct Modeling Strategies for Gene and Gene-specific Treatment Effects in Hierarchical Models for Microarray Data. Annals of Applied Statistics. 
HIERARCHICAL MODELING STRATEGIES FOR GENE AND TREATMENT EFFECTS 11

List of Figures. $\mathrm{N}^{3} \mathrm{GV}$ model estimates of $\sigma_{\gamma}^{2}$ and $\sigma_{\tau}^{2}$ for simulated and real microarry data . . . . . . . . . . 12

2 Observed proportion of null genes vs. ePPEE for simulated data . 13

3 Comparison across methods of empirical ePPDE CDFs for simulations from HIV data (top) and $\mathrm{LN}^{3} \mathrm{GV}$ model (bottom) . . . . . 14

4 ROC curves for simulations from $\mathrm{HIV}$ data (top) and $\mathrm{LN}^{3} \mathrm{GV}$ model

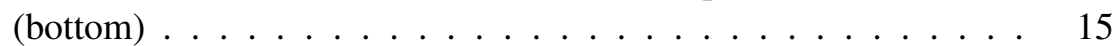

5 ROC curves for simulations from $\mathrm{LN}^{3}$ (top) and LNNMV (bottom) models . . . . . . . . . . . . . . . . 16

6 ROC curves for simulations from GG (top) and LNN (bottom) models 17 


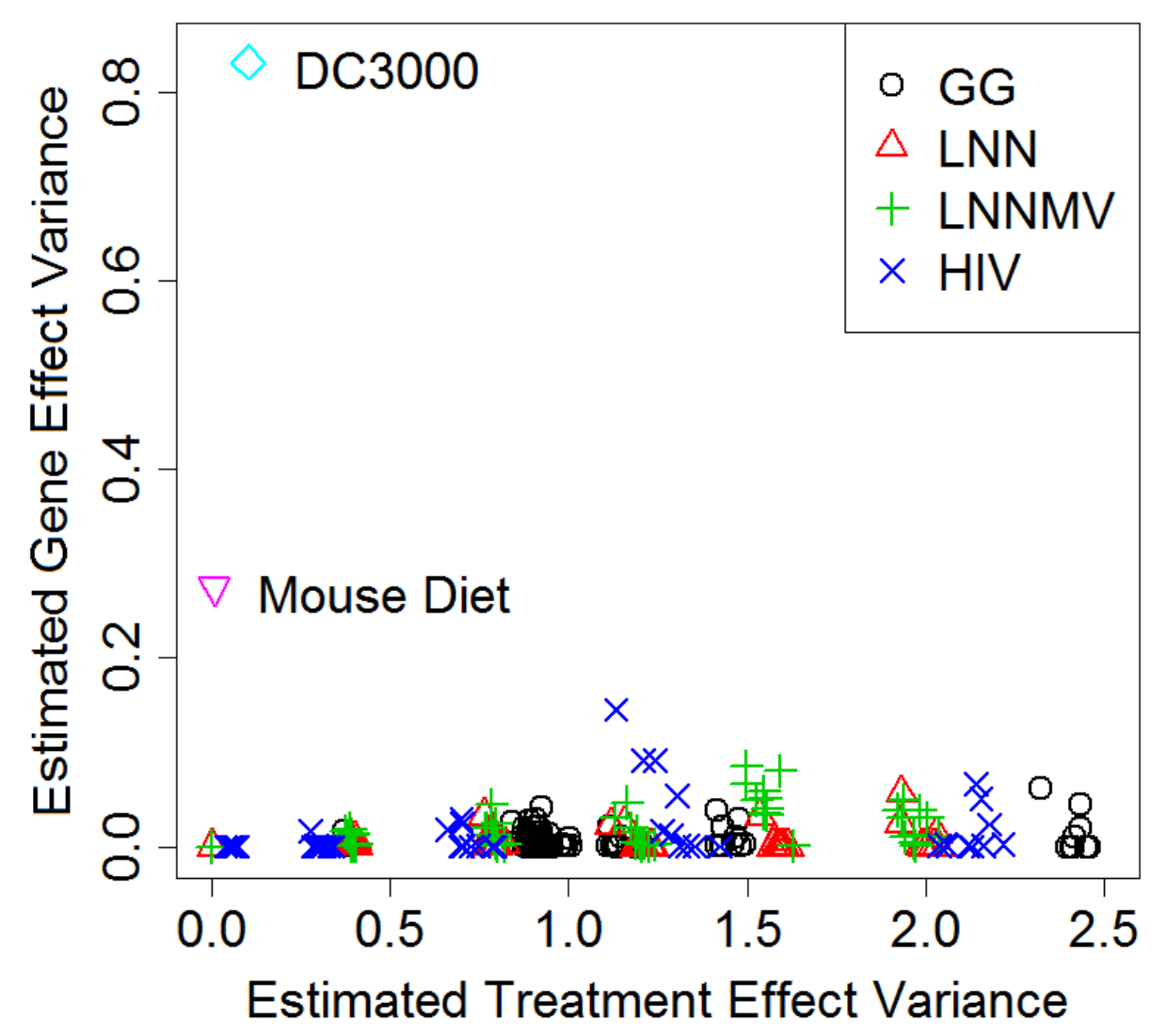

FIG 1. $L N^{3} G V$ model estimates of $\sigma_{\gamma}^{2}$ and $\sigma_{\tau}^{2}$ for simulated and real microarry data 


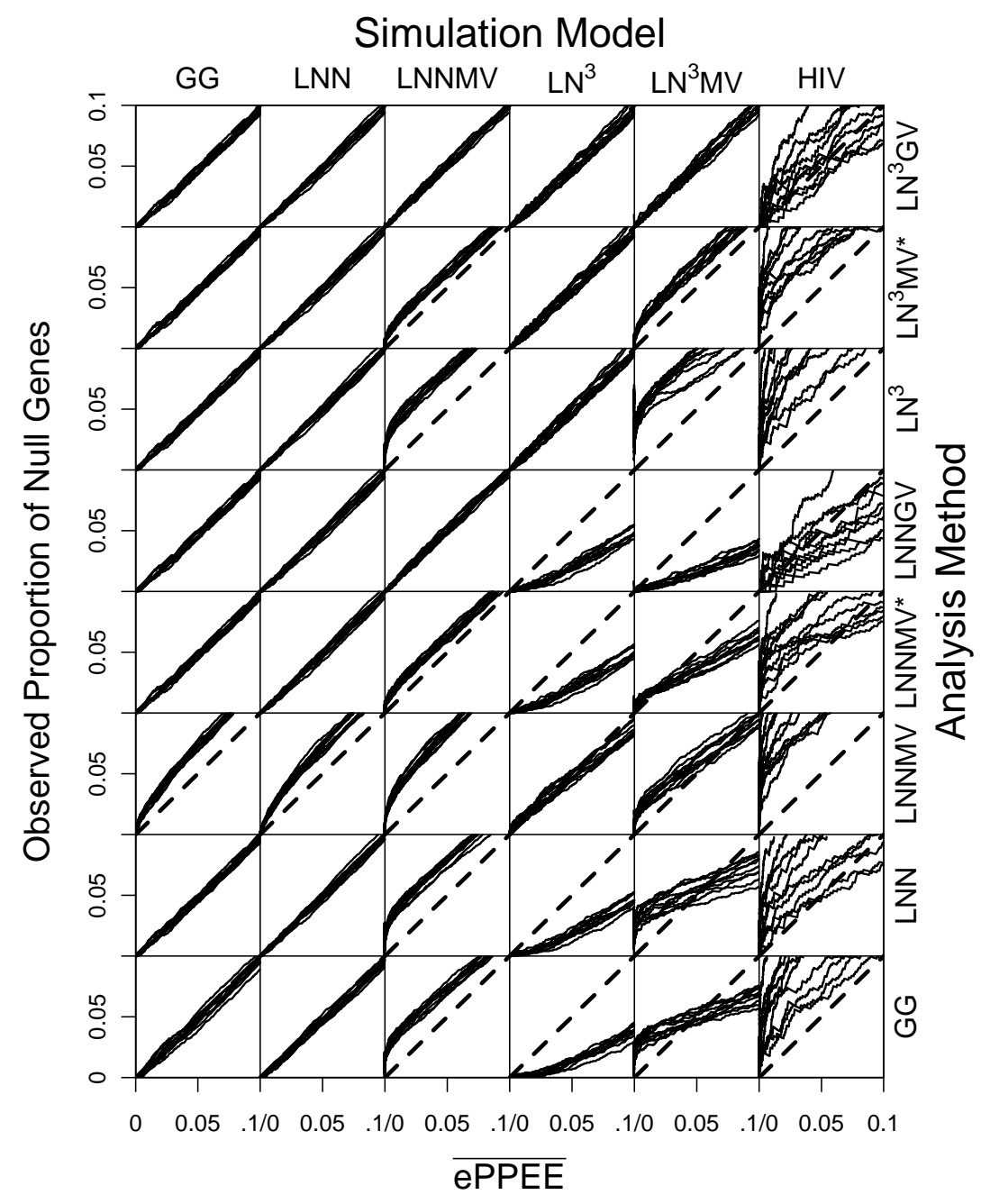

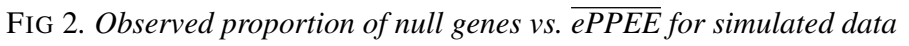



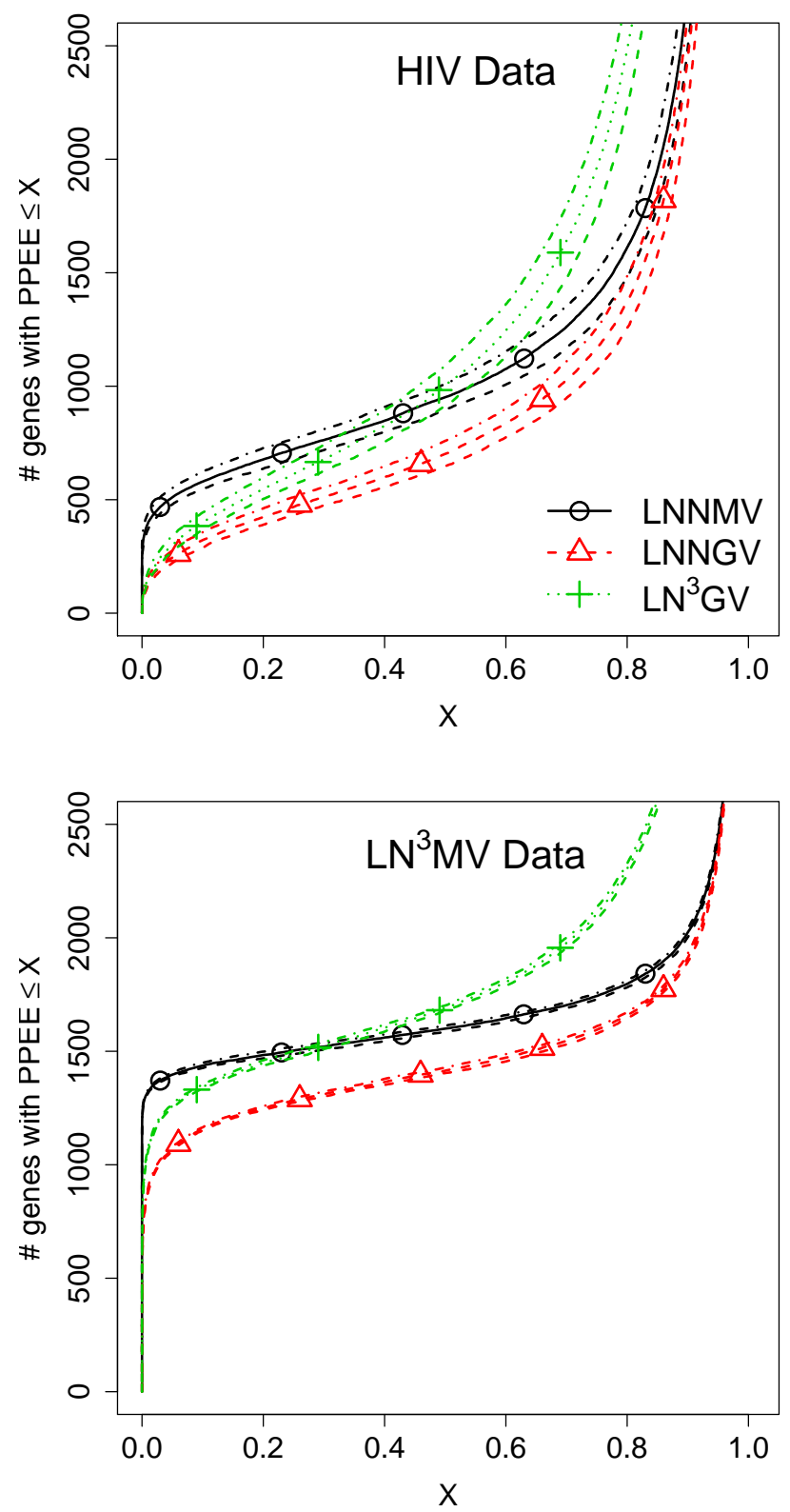

FIG 3. Comparison across methods of empirical ePPDE CDFs for simulations from HIV data (top) and $L N^{3} G V$ model (bottom) 

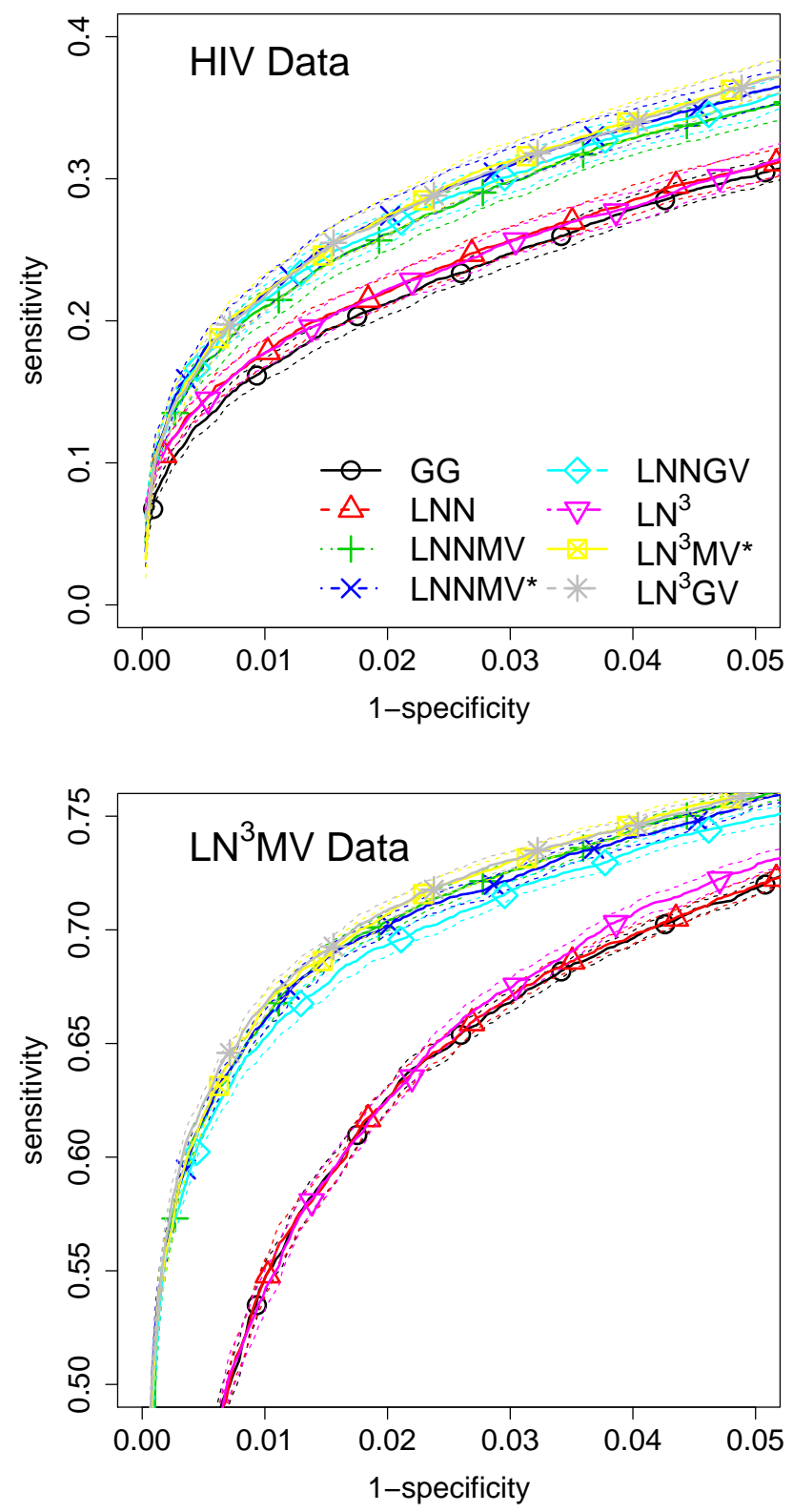

FIG 4. ROC curves for simulations from HIV data (top) and $L N^{3} G V$ model (bottom) 

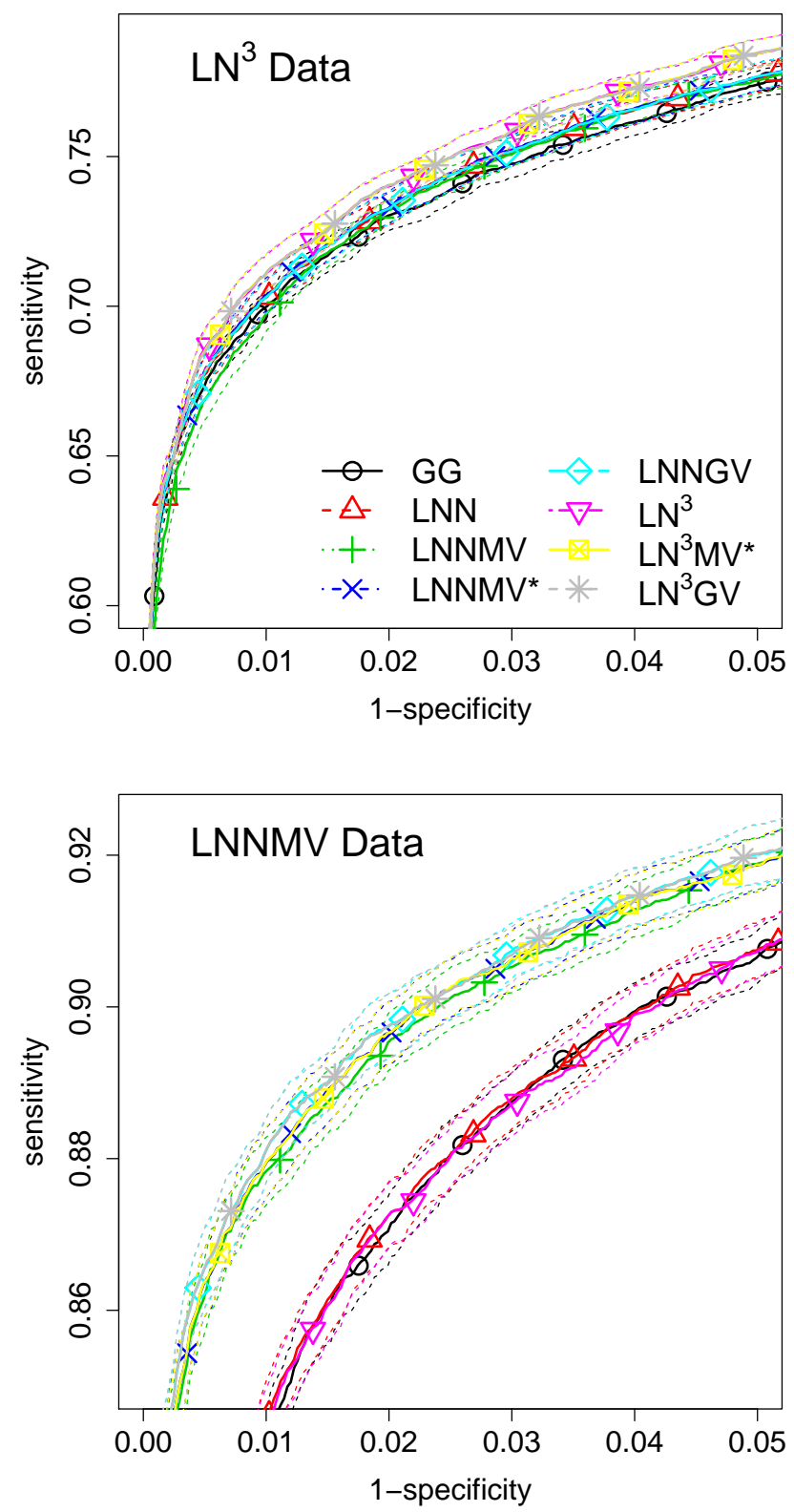

FIG 5. ROC curves for simulations from $L N^{3}$ (top) and LNNMV (bottom) models 

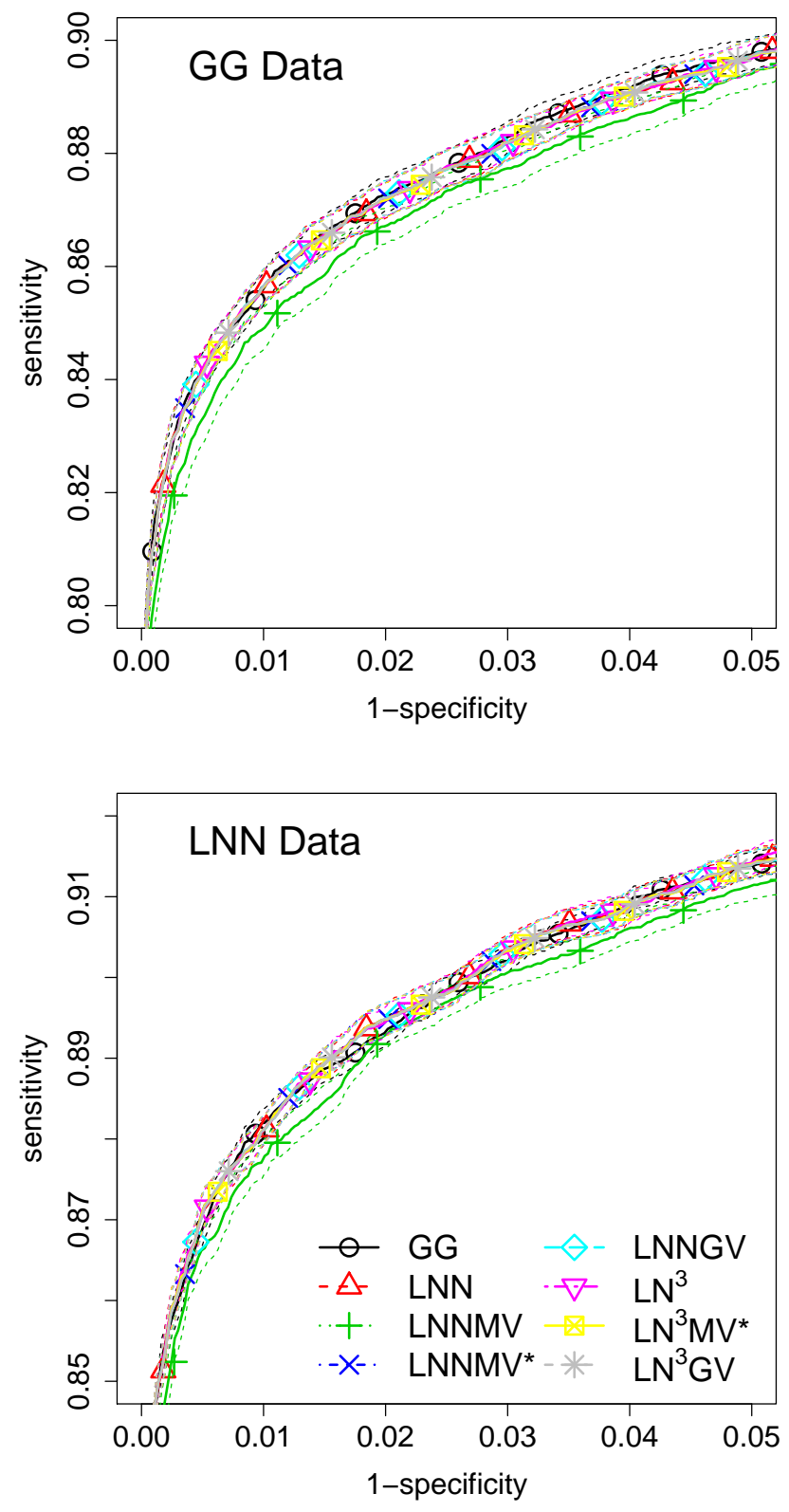

FIG 6. ROC curves for simulations from GG (top) and LNN (bottom) models 